\title{
Indications of a Large Fraction of Spectroscopic Binaries Among Nuclei of Planetary Nebulae
}

\author{
Orsola De Marco ${ }^{1}$, Howard E. Bond ${ }^{2}$, Dianne Harmer ${ }^{3}$, and Andrew J. Fleming ${ }^{4}$
}

\begin{abstract}
Previous work indicates that about $10 \%$ of planetary-nebula nuclei $(\mathrm{PNNi})$ are photometrically variable short-period binaries with periods of hours to a few days. These systems have most likely descended from common-envelope (CE) interactions in initially much wider binaries. Population-synthesis studies suggest that these very close pairs could be the short-period tail of a much larger postCE binary population with periods of up to a few months. We have initiated a radial-velocity (RV) survey of PNNi with the WIYN 3.5-m telescope and Hydra spectrograph, which is aimed at discovering these intermediate-period binaries. We present initial results showing that 10 out of 11 well-observed PNNi have variable RVs, suggesting that a significant binary population may be present. However, further observations are required because we have as yet been unable to fit our sparse measurements with definite orbital periods, and because some of the RV variability might be due to variations in the stellar winds of some of our PNNi.
\end{abstract}

Subject headings: techniques: radial velocities - stars: AGB and post-AGB binaries: spectroscopic - white dwarfs - planetary nebulae: general

\section{Introduction}

There is reason to think that many of the central stars in planetary nebulae $(\mathrm{PNe})$ may be close binaries. The evidence leading to this suggestion includes the following:

\footnotetext{
${ }^{1}$ Department of Astrophysics, American Museum of Natural History, Central Park West at 79th St., New York, NY 10024; orsola@amnh.org

${ }^{2}$ Space Telescope Science Institute, 3700 San Martin Drive, Baltimore, MD 21218; bond@stsci.edu

${ }^{3}$ Kitt Peak National Observatory, National Optical Astronomy Observatories, P.O. Box 26732, Tucson, AZ 85719; diharmer@noao.edu

${ }^{4}$ REU fellow, summer 2003, American Museum of Natural History; Department of Physics, Michigan Technological University, 1400 Townsend Drive, Houghton, MI 49931; ajflemin@mtu.edu
} 
(1) Photometric monitoring has revealed that about $10 \%$ of planetary-nebula nuclei (PNNi) are very close binaries, with periods of a few hours up to a few days (e.g., Bond \& Livio 1990; Bond 2000). These close binaries in PNe provide direct evidence for the occurrence of a common-envelope (CE) interaction (Paczynski 1976; Sandquist et al. 1998), in which one component of a binary evolves to giant dimensions, and then engulfs a mainsequence companion; the ensuing spiral-down of the orbit ejects the $\mathrm{CE}$ and exposes the hot core of the red giant, leaving a close binary inside a photoionized PN.

(2) Theoretical studies of the evolution of binary populations (e.g. Yungelson et al. 1993, Han et al. 1995), predict the orbital period distribution of binary stars inside PNe to be a strong function of the efficiency, $\alpha_{\mathrm{CE}}$, with which the orbital energy of the original system goes into ejecting material from the CE. However, a recent study (O'Brien et al. 2001 ) of the post-CE eclipsing binary V471 Tauri indicates that $\alpha_{\mathrm{CE}} \approx 0.1$, for this one object. Fig. 3b of Yungelson et al. then predicts, if this value of $\alpha_{\mathrm{CE}}$ is applicable to most $\mathrm{CE}$ interactions, that the orbital periods of binaries in PNe should be distributed roughly evenly over the range $0.3-30$ days. Since $10 \%$ of PNNi are already known to be binaries lying in the short-period tail that is detectable photometrically, these results suggest that a large fraction of PNNi could be longer-period binaries.

(3) A large majority of PNe have highly non-spherical shapes, including numerous extreme cases of strongly bipolar or axisymmetric morphologies (e.g., Zuckerman \& Aller 1986; Soker 1997). The simplest explanation for these shapes would be that most PNe have been ejected through CE interactions, or that the PN ejection process has at least been affected by other phenomena directly related to the presence of a companion star (e.g., tidal spinup and/or dynamo generation of magnetic fields).

The strongest empirical test of this hypothesis of a large binary fraction would be to search for the expected population of binary PNNi with periods too long to be detected from photometric variability, but detectable through radial-velocity (RV) variations. Knowledge of the overall period distribution of binary PNNi would provide strong constraints on (a) the binary properties of the parent AGB population, and (b) the typical value of $\alpha_{\mathrm{CE}}$, a quantity needed to predict the properties of other post-CE systems, including cataclysmic variables, low-mass X-ray binaries, and the progenitors of Type Ia supernovae.

In this Letter we report initial results of a RV survey of PNNi, designed to detect variability on timescales of a few days up to a few months, with velocity amplitudes down to a few $\mathrm{km} \mathrm{s}^{-1}$. 


\section{Target Selection and Observations}

Our spectroscopic data were taken at the 3.5-m WIYN telescope at Kitt Peak National Observatory between 2002 August and 2003 September. It is well known (e.g., Kennicutt, Freedman, \& Mould 1995) that for optimum sampling of unknown periods, the observations should be spaced over an interval at least as long as the longest expected period, with the intervals between successive observations increasing according to a power series.

Since we wished to search for periods of a few days up to about two months, this scheduling requirement dictated use of a spectrograph that is easily available on the telescope without major instrument changes, making runs as short as one night possible. The Hydra fiber-optics bench-mounted spectrograph (Barden \& Armandroff 1995), which can be put into use very quickly on the WIYN telescope by rotating the Nasmyth tertiary mirror, was thus ideal for meeting our scheduling needs. We were awarded 8 nights each in three successive semesters, with each semester's individual nights spaced nearly ideally as outlined above. Unfortunately, however, we encountered unusually bad weather - not to mention a nearby forest fire - during all three semesters, so that the actual sampling that we achieved was very far from ideal.

We used the Hydra spectrograph with fibers that project to a 2".0 diameter on the sky; this diameter is near optimal for collecting most of the starlight in typical seeing, while giving little degradation of the spectral resolution, and permitting minimal contamination from the surrounding nebulae. A 1200 groove $\mathrm{mm}^{-1}$ grating, combined with the "Red Bench" camera and the T2KC CCD detector, provided a dispersion of $0.33 \AA$ pixel $^{-1}$ and a wavelength coverage of 4050-4730 $\AA$. The FWHM of comparison lines was typically $0.60 \AA$. We generally obtained a Cu-Ar comparison lamp exposure after every three stellar exposures, which was adequate because of the high stability of the bench spectrograph.

Our goal was to conduct a RV survey of a representative selection of PNNi. However, as is well known, many PNNi have significant stellar winds, raising the danger that shorttimescale variations in mass-loss rate could give rise to spurious apparent velocity variations. (Note, for example, that virtually all PNNi with obvious P Cygni profiles in the ultraviolet are observed to vary photometrically on short timescales-Bond \& Ciardullo 1989; Handler et al. 1997.) In order to maximize the probability of measuring center-of-mass motions of

the stars, we drew up a list of targets likely to have low mass-loss rates and optical spectra dominated by photospheric absorption lines. These included stars classified as type O (e.g., by Heap 1977), sdO, B, or sdB by various authors. In order to minimize exposure times, we favored stars brighter than about 14th visual magnitude. Several candidate stars were observed, but found to have few or no usable absorption lines, and were thus dropped from our program. At this writing, we have obtained useful spectra of $11 \mathrm{PNNi}$ on at least 4, and 
up to 16 , different nights.

In addition to the PNNi, we obtained spectra on most nights of the well-known sdO spectrophotometric standard star BD $+28^{\circ} 4211$. This star has a spectrum similar to those of some of our program stars, but is not known to be surrounded by a $\mathrm{PN}^{5}$. To the best of our knowledge this star has not been reported to have a variable RV, and thus it should serve to monitor the stability of our velocity measurements for the program stars.

\section{Data Reduction and Analysis}

We reduced our spectra with NOAO IRAF ${ }^{6}$ software. The dohydra task was used to flat-field, extract, and wavelength-calibrate the spectra. Excellent fits to the wavelength dispersion curve were obtained, with RMS scatter of about $0.03 \AA$.

We measured RV shifts between normalized pairs of spectra using the cross-correlation technique implemented by the IRAF task fxcor. For each star in our sample, we initially selected the spectrum with the highest signal-to-noise ratio (SNR) as the template, and cross-correlated it with the other spectra taken at different epochs. We then used these initial RV estimates to shift each spectrum to the velocity of the template, and created a weighted average of all of them. This averaged spectrum, with typical SNR $\geq 100$, was then used as the final template to determine the relative RV shifts between it and each of the spectra. In the present work, we have not attempted to place the RVs on an absolute velocity scale.

The fxcor task calculates the velocity shift between two spectra by fitting the correlation with a user-selected function. Most of our PNNi have a variety of line profiles, including pressure-broadened Balmer lines as well as narrower lines from metallic species. A parabola was finally adopted as the best fitting function.

We regard the errors in our relative velocities as arising from two sources. One is the random velocity scatter due to errors in the dispersion fit, mechanical drifts in the spectrograph, and other less easily specified effects. To estimate this scatter, we measured

\footnotetext{
${ }^{5}$ As noted by Zanin \& Weinberger (1997), the Palomar Sky Survey shows some diffuse nebulosity with an angular extent of several degrees in the vicinity of $\mathrm{BD}+28^{\circ} 4211$. If actually associated with the star, this nebulosity would have a physical scale much larger than any other known PN.

${ }^{6}$ IRAF is distributed by the National Optical Astronomy Observatories, which are operated by the Association of Universities for Research in Astronomy, Inc., under cooperative agreement with the National Science Foundation.
} 
the relative velocities of the strong nebular [O III] $4363 \AA$ emission line of NGC 6891, based on 17 spectra. Such a sharp nebular line is expected to have a constant RV. The RMS scatter of the measured relative velocities was found to be $2.1 \mathrm{~km} \mathrm{~s}^{-1}$, which we take as a measure of the random velocity errors affecting all of our measurements (note that this is closely similar to the velocity scatter expected from the $0.03 \AA \simeq 2.0 \mathrm{~km} \mathrm{~s}^{-1}$ dispersion of our comparison-line fits).

In addition, there is a contribution from the errors of the individual absorption-line cross-correlation fits due to effects such as photon noise and the shapes and widths of the absorption-line profiles. The fxcor task reports a random error for each velocity shift based on the error in fitting a parabola to the cross-correlation function. For a sharp, strong emission line such as [O III] $4363 \AA$ in NGC 6891, the error reported by fxcor is negligible $\left(<0.2 \mathrm{~km} \mathrm{~s}^{-1}\right)$ compared to the night-to-night scatter. For the absorption-line RVs of our program stars, we found that the fxcor errors are also less than the random night-to-night error estimated above, except for spectra with low SNR, or with especially broad or shallow absorption lines. In our analysis below, we combine in quadrature the random error estimate of $2.1 \mathrm{~km} \mathrm{~s}^{-1}$ with the random error from fxcor, in order to estimate the overall error of each RV measurement.

The stellar absorption lines most commonly encountered in PNNi in our spectral range are $\mathrm{H} \delta$ and $\mathrm{H} \gamma$ (often contaminated by nebular emission), as well as lines of He I, He II, C III, C IV, N III, and N V. When determining the cross correlation using stellar absorption lines, we selected spectral windows so as to exclude the nebular emission lines in those objects with strong superposed emission.

\section{Results and Discussion}

In Table 1 we present the list of stars for which we have obtained at least 4 good (continuum SNR $\geq 20$ ) spectroscopic observations. In columns (5), (6), and (7), we list the number of times each star was observed, $n$, the standard deviation, $\sigma$, of the ensemble of relative RV measurements, and the average error of an individual RV measurement. Each star was observed once per night, on as many nights as the weather and sidereal time permitted.

The question whether a collection of RV measurements is consistent with the hypothesis of a constant velocity is a classical problem, discussed in detail for example by Trumpler \& Weaver (1953). These authors recommend a standard $\chi^{2}$ calculation, from which the reduced value, $\chi^{2} /(n-1)$ (given in Table 1, column [8]), leads to an estimate of the probability that the star's RV is variable (column [9]; e.g., Press et al. 1986). 
Table 1 shows the startling conclusion that 10 out of the 11 PNNi that we have observed have variable velocities. Reassuringly, our control star, BD $+28^{\circ} 4211$, has a constant RV according to our statistical test (i.e., it has only a $24 \%$ probability of variability). (As Table 1 shows, the individual RV errors for BD $+28^{\circ} 4211$ are relatively large, averaging $3.3 \mathrm{~km} \mathrm{~s}^{-1}$. This is due to the relatively high gravity of this star, which gives it broader lines than our typical PNNi; hence the cross-correlation function is broader, with an attendant larger error in the velocity shifts.)

Are these RV variations due to motion in a binary system, or to some other phenomenon (e.g., stellar-wind variations that modulate absorption-line profiles in a way that mimics velocity variability)? Detection of a clearly periodic velocity variation would provide strong support for binary motion. Unfortunately, due to the poor weather that we encountered, we have only a handful of observations of each star spaced over more than a one-year interval. Most of our stars appear to show RV variations on timescales as short as 1 day, suggesting that their periods, if they are binaries, are relatively short. Thus our sampling is extremely non-optimal for period searching. We have used the Lafler \& Kinman (1965) periodogram to search for periodic signals, but due to the severe aliasing it is not possible to find a definite period for any of our stars; a very wide variety of periods can yield more-or-less smooth RV curves, but none of them stand out clearly.

In order to investigate further for the presence of winds, we examined UV spectra of our targets obtained with the International Ultraviolet Explorer (IUE) satellite, using the data archive at the Space Telescope Science Institute ${ }^{7}$. All 12 of our targets, including $\mathrm{BD}+28^{\circ} 4211$, have been observed with IUE, but only five of them (IC 4593, NGC 6210, NGC 6891, A 78, and Sa 4-1) show P Cygni profiles in the UV. However, most optical absorption lines are much less affected by winds than the UV resonance lines. Moreover, asymmetric profiles would typically be introduced for lines formed in a wind, but we did not observe any unusual profiles in the lines measured for RV (note that fxcor directly displays the correlation function, which has the same shape as the typical stellar absorption lines), nor any obvious time variations in the line profiles. Thus, although we cannot absolutely exclude that the RVs of these five targets are affected by wind phenomena, we believe it to be unlikely; and in any case, we find it hard to believe that the variations of the other five variables are due to such a phenomenon.

We thus have a tantalizing suggestion that the binary fraction among PNNi may be very high. As noted above, photometric monitoring has indicated that about $10 \%$ of randomly selected PNNi are short-period (hours to a few days) binaries. The RV results presented in

\footnotetext{
${ }^{7}$ http://archive.stsci.edu/iue/index.html
} 
this paper now suggest that an even larger fraction (which may well exceed $50 \%$, and may approach 100\%) of random PNNi may be spectroscopic binaries of intermediate periods. Our results appear to be consistent with those presented recently by Pollacco (2003), who finds, in a somewhat less sensitive RV survey, that $57 \%$ of a sample of 23 PNNi have variable RVs.

We plan to continue our monitoring of northern-hemisphere targets, and we are currently analyzing RV data for about 30 southern-hemisphere PNNi. Such observations will greatly clarify the situation. At present, it has become increasingly plausible that binary-star ejection is a major formation channel for planetary nebulae.

\section{APPENDIX}

Comments on individual objects:

PHL 932 is almost unique among PNNi in being classified as a sdB star. Its effective temperature and surface gravity (Napiwotzki 1999) suggest it could be a post-red giant in a binary system that underwent a CE episode (Mendez et al. 1988; Iben \& Tutukov 1993). No RV variations greater than $2 \mathrm{~km} \mathrm{~s}^{-1}$ were detected over an interval of 6 days by Wade (2001). Our claim of RV variability rests largely on one outlying velocity measurement, differing from the mean of the remaining 8 observations by $-8.9 \pm 2.5 \mathrm{~km} \mathrm{~s}^{-1}$; this suggests that, if the star is a spectroscopic binary, its eccentricity is high, which would be surprising for a post-CE system.

$\mathrm{BD}+33^{\circ} 2642$ is an extremely well-observed spectrophotometric standard star, so it is a surprise that its surrounding faint PN was not discovered until 10 years ago (Napiwotzki 1993). Our RV measurements show a range of $15.3 \mathrm{~km} \mathrm{~s}^{-1}$, well in excess of the small errors for this bright star with many usable photospheric absorption lines.

IC 4593, NGC 6210, NGC 6891. Our measurements of these three rather similar objects rely on absorption lines of He II at 4200 and $4541 \AA$, as well as the wings of the Balmer lines (the cores are contaminated with nebular emission). We find definite RV variability in all three, with total ranges of $36.4,16.7$, and $15.6 \mathrm{~km} \mathrm{~s}^{-1}$, respectively. However, the nucleus of IC 4593 is variable photometrically on a timescale of hours (Bond \& Ciardullo 1989), has P Cygni profiles in the UV, and shows short-timescale variations in its optical (Mendez, Herrero, \& Manchado 1990) and UV (Patriarchi \& Perinotto 1995) emission features. Mendez et al. also reported optical absorption-line RV variations in IC 4593 similar to those we report here. The other two PNNi likewise show P Cygni profiles in the UV. We therefore cannot completely rule out that the optical RV variability that we measured is due to variations in the stellar-wind mass loss.

IRAS $\mathbf{1 9 1 2 7 + 1 7 1 7}$ was suspected of binarity by Whitelock \& Menzies (1986), because 
the contrast between the high excitation of the PN with the relatively cool temperature of the B9 central star suggested that the ionization source is an optically inconspicuous hot companion. We find strong evidence for RV variability, with a total range of $33.0 \mathrm{~km} \mathrm{~s}^{-1}$.

LS IV $-\mathbf{1 2}^{\circ} \mathbf{1 1 1}$, originally discovered in the Case-Hamburg survey for luminous earlytype stars, was re-classified as a post-AGB star embedded in a young PN by Conlon et al. (1993). Arkhipova et al. (2002) have reported photometric variations, and Ryans et al. (2003) suspected RV variability on the basis of 3 velocity measurements. We confirm definite RV variability, with a total range of $39.0 \mathrm{~km} \mathrm{~s}^{-1}$.

M 1-77, M 2-54 have both been reported to be photometric variables on timescales of hours (Handler 1995, 1999). We find definite RV variability for both PNNi (total ranges of 32.8 and $49.0 \mathrm{~km} \mathrm{~s}^{-1}$, respectively). Although the photometric variability might suggest a connection with the wind variability discussed above for IC 4593 and similar objects, our spectra show a wealth of sharp absorption lines, and IUE spectra show no P Cygni profiles. We thus believe there is a high probability that both stars are spectroscopic binaries, although the non-periodic photometric variability would then be left unexplained.

A 78 is a prototypical born-again PNN (Iben et al. 1983; Jacoby \& Ford 1983). Like IC 4593, NGC 6210, and NGC 6891, it has pronounced P Cygni profiles in the UV, so the RV variations that we find (total range $23.1 \mathrm{~km} \mathrm{~s}^{-1}$ ) might conceivably be attributable to wind variability.

Sa 4-1 has a high-velocity wind seen as blue-shifted absorption in high-dispersion IUE spectra, with relatively weak emission near zero velocity (Feibelman \& Bruhweiler 1989), putting it at risk of wind variability. Our 4 spectra, however, show only marginal evidence for RV variation.

We are thankful for the terrific effort of the WIYN/NOAO team in supporting our project, in particular WIYN Observatory Director George Jacoby, telescope operators Gene McDougall, George Will, Doug Williams, and Hillary Mathis, and instrument specialist Chuck Corson. We thank John Glaspey for carrying out the difficult task of meeting our exacting telescope scheduling requirements. Don Pollacco provided useful information in advance of publication. OD is grateful to Janet Jeppson Asimov for financial support.

\section{REFERENCES}

Acker, A., Ochsenbein, F., Stenholm, B., Tylenda, R., Marcout, J., \& Schohn, C. 1992, Strasbourg-ESO Catalogue of Galactic Planetary Nebulae (Garching, ESO) 
Arkhipova, V.P., Ikonnikova, N.P., Noskova, R.I., \& Komissarova, G.V. 2002, Astronomy Letters, 28, 257

Barden, S.C., \& Armandroff, T., 1995, Fiber Optics in Astronomical Applications, S. Barden, SPIE Vol. 2476, 56

Bond, H.E. 2000, ASP Conf. Ser. 199: Asymmetrical Planetary Nebulae II: From Origins to Microstructures, 115

Bond, H.E., \& Ciardullo, R. 1989, IAU Colloq. 114: White Dwarfs, 473

Bond, H.E., \& Livio, M. 1990, ApJ, 355, 568

Conlon, E.S., Dufton, P.L., McCausland, R.J.H., \& Keenan, F. P. 1993, ApJ, 408, 593

Feibelman W.A., \& Bruhweiler, F.C. 1989, ApJ, 347, 901

Han, Z., Podsiadlowski, P., Eggleton, P.P. 1995, MNRAS, 272, 800

Handler, G. 1995, Information Bulletin on Variable Stars, 4244, 1

Handler, G. et al. 1997, A\&A, 320, 125

Handler, G. 1999, A\&AS, 135, 493

Heap, S.R. 1977, ApJ, 215, 609

Iben, I., Jr., \& Tutukov, A. V. 1993, ApJ, 418, 343

Iben, I., Jr., Kaler, J.B., Truran, J.W., \& Renzini, A. 1983, ApJ, 264, 605

Jacoby, G.H., \& Ford, H.C. 1983, ApJ, 266, 298

Kennicutt, R. C., Freedman, W. L., \& Mould, J. R. 1995, AJ, 110, 1476

Lafler, J., \& Kinman, T.D. 1967, ApJS, 11, 216

Mendez, R. H., Kudritzki, R. P., Groth, H. G., Husfeld, D., \& Herrero, A. 1988, A\&A, 197, L25

Mendez, R.H., Herrero, A., \& Manchado, A. 1990, A\&A, 229, 152

Napiwotzki, R. 1993, Acta Astronomica, 43, 415

Napiwotzki, R. 1999, A\&A, 350, 101 
O’Brien, M.S., Bond, H.E., \& Sion, E.M. 2001, ApJ, 563, 971

Patriarchi, P., \& Perinotto, M. 1995, A\&AS, 110, 353

Paczynski, B. 1976, IAU Symp. 73: Structure and Evolution of Close Binary Systems, 75

Pollacco, D.L., 2003, Asymmetric Planetary Nebulae III, in press

Press, W. H., Flannery, B. P., Teukolsky, S. A., \& Vetterling, W. T. 1986, Numerical Recipes (Cambridge: Cambridge University Press), 165

Ryans, R.S.I., Dufton, P.L., Mooney, C.J., Rolleston, W.R.J., Keenan, F.P., Hubeny, I., \& Lanz T. 2003, A\&A, 401, 1119

Sandquist, E.L., Taam, R.E., Chen, X., Bodenheimer, P., \& Burkert, A., 1998, ApJ, 500, 909

Soker, N. 1997, ApJS, 112, 487

Trumpler, R. J., \& Weaver, H. F. 1953, Statistical Astronomy (New York: Dover Publications), 205

Wade, R. A. 2001, ASP Conf. Ser. 226: 12th European Workshop on White Dwarfs, 199

Whitelock, P. A., \& Menzies, J. W. 1986, MNRAS, 223, 497

Yungelson, L.R., Tutukov, A.V., \& Livio, M. 1993, ApJ, 418, 794

Zanin, C., \& Weinberger, R. 1997, IAU Symp. 180: Planetary Nebulae, 180, 290

Zuckerman, B., \& Aller, L.H., 1986, ApJ, 301, 772 
Table 1. Target list and Radial-Velocity Measurements

\begin{tabular}{|c|c|c|c|c|c|c|c|c|}
\hline Star & PN G & $\begin{array}{c}V \\
\text { mag }\end{array}$ & $\begin{array}{l}\text { Sp. } \\
\text { Type }\end{array}$ & $n$ & $\begin{array}{c}\sigma \\
\left(\mathrm{km} \mathrm{s}^{-1}\right)\end{array}$ & $\begin{array}{l}\text { Error of } 1 \text { obs } \\
\quad\left(\mathrm{km} \mathrm{s}^{-1}\right)\end{array}$ & $\chi^{2}$ & $\begin{array}{l}P \\
\text { var }\end{array}$ \\
\hline \multicolumn{9}{|c|}{$\mathrm{RV}$ variables } \\
\hline PHL 932 & $125.9-47.0$ & 12.1 & hg $\mathrm{O}(\mathrm{H})$ & 9 & 3.8 & 2.6 & 2.28 & 0.98 \\
\hline $\mathrm{BD}+33^{\circ} 2642$ & $052.7+50.7$ & 10.8 & B2 IVp & 14 & 3.7 & 2.3 & 2.62 & 1.00 \\
\hline IC 4593 & $025.3+40.8$ & 11.2 & $\mathrm{O} 5 \mathrm{f}(\mathrm{H})$ & 8 & 11.9 & 3.0 & 18.19 & 1.00 \\
\hline NGC 6210 & $043.1+37.7$ & 12.7 & $\mathrm{O}(\mathrm{H})$ & 6 & 5.8 & 2.4 & 6.67 & 1.00 \\
\hline IRAS $19127+1717$ & $051.0+02.8$ & 13.4 & B9 V & 12 & 9.5 & 3.1 & 9.94 & 1.00 \\
\hline LS IV $-12^{\circ} 111$ & $029.1-21.2$ & 11.4 & B1 Ibe & 15 & 12.1 & 2.3 & 28.78 & 1.00 \\
\hline NGC 6891 & $054.1-12.1$ & 12.4 & Of $(H)$ & 16 & 4.6 & 2.6 & 3.16 & 1.00 \\
\hline M 1-77 & $089.3-02.2$ & 12.1 & $\mathrm{OB}$ & 15 & 9.5 & 2.5 & 15.43 & 1.00 \\
\hline A 78 & $081.2-14.9$ & 13.2 & Of/WR $(\mathrm{C})$ & 11 & 5.1 & 2.6 & 3.99 & 1.00 \\
\hline M 2-54 & $104.8-06.7$ & 12.1 & $\mathrm{~B}$ & 15 & 11.8 & 2.6 & 21.30 & 1.00 \\
\hline \multicolumn{9}{|c|}{ Possible RV variable } \\
\hline Sa $4-1$ & $075.7+35.8$ & 14.3 & $\mathrm{O}(\mathrm{H})$ & 4 & 2.4 & 2.4 & 1.25 & 0.71 \\
\hline \multicolumn{9}{|c|}{ Control star } \\
\hline $\mathrm{BD}+28^{\circ} 4211$ & $\ldots$ & 10.5 & $\mathrm{sdO}$ & 14 & 2.9 & 3.3 & 0.70 & 0.24 \\
\hline
\end{tabular}

Note. - Cols. 1 and 2: target name and designation in PN G nomenclature; cols. 3 and 4: $V$ magnitude and spectral type, from Acker et al. (1992) or SIMBAD database; col. 5: number of spectroscopic observations; col. 6: weighted RMS scatter of our RV measurements; col. 7: weighted mean error of one RV observation; col. 8: $\chi^{2}$ per degree of freedom; col. 9: probability that the star's RV is variable. 